\title{
Editorial
}

\section{Towards an inclusive, responsible and sustainable open access model}

\author{
Pietro Baroni and Bart Verheij \\ Editors-in-Chief
}

In recent years the world of scientific publishing has rapidly evolved into a complex ecosystem, witnessing the coexistence of a variety of publishing venues, which widely differ in several key aspects like breadth of topics, intended audience, reviewing process, volume of yearly published material, and subscription and/or open access fees.

In this heterogeneous context, Argument \& Computation stands as a community-driven journal, whose mission is serving the dissemination of high-quality research results produced by the worldwide community of scholars interested in all aspects of computational argumentation, a conceptually broad but well identified and focused interdisciplinary topic.

To fully accomplish its mission our journal (and, we would say, any scientific journal) needs to be inclusive with respect to both authors and readers. The authors should not meet obstacles of any kind, in particular of economic nature, to the submission and publication of contributions which meet the journal's quality standards. These contributions should then be easily available to any interested reader.

To achieve these goals the journal needs in turn to implement a sustainable policy, putting into place the technical and professional resources needed to support all the phases of the publishing process, from submission and review management to the production and long-term availability of the final versions, while covering the relevant costs.

Since 2016 the journal has achieved its inclusiveness goals by applying a full open-access policy with no fees for authors or readers, thanks to the generous support of our publisher, IOS Press, ensuring the consolidation of the journal in the delicate phase of transition from a previous publisher. It was agreed from the beginning that this particularly favorable arrangement could only be temporary and that, in the long term, a balanced way to sustain open access had to be identified. After six successful years of journal development and consolidation, the time has come to make a move forward in this direction.

To ensure an open and informed decision process on this key step in the journal's life, a consultation about publication policies has been conducted by proposing a questionnaire to the editorial board members and, more broadly, to the community members, through the COMMA mailing list. The outcomes of the questionnaire have been discussed by the Editors-in-Chief both with the COMMA steering committee (thereby representing the interests of the computational argumentation community) and with the publisher. It was agreed that the goal of preserving inclusiveness should have the highest priority and it has been observed that several commonly adopted publication policies, involving a mandatory open access fee, would be incompatible with the full satisfaction of this goal. 
For this reason the decision has been made to adopt a publication model based on responsible voluntary support by the community. Simply stated, in this model authors are offered the option to sustain the journal by paying a voluntary open access fee, as a free choice if feasible for them. This can for instance be the case when paid open access is covered by an institutional arrangement, or when there are applicable project funds. The idea is that all potential authors will still be completely free to submit and publish their papers with no obligations (financial or administrative) while at the same time all the authors will be free to decide whether to pay an open access fee for their accepted papers.

In the long term, the continuation of the journal under this policy will rely on the responsibility of those authors and institutions who, having access to adequate funding resources for open access publications, are available to support the openness of the journal for the benefit of those colleagues who have to cope with less favorable situations. The editors and the publisher will be monitoring the results of this policy.

To complete the picture, coverage by several agreements at national and international level and a range of waiver conditions are also considered. For more detailed information please refer to the Author Guidelines.

We are confident that this model, by combining the values of inclusiveness and responsibility, will be successful in ensuring the sustainability and future development of the journal and count on the community support to achieve this goal.

Pietro Baroni

Bart Verheij

Editors-in-Chief 